\title{
Evolution of the vortex and the asymmetrical parts of orbital angular momentum in separable first-order optical systems
}

\author{
Tatiana Alieva \\ Universidad Complutense de Madrid, Facultad de Ciencias Físicas, Ciudad Universitaria s/n, Madrid 28040, Spain \\ Martin J. Bastiaans \\ Technische Universiteit Eindhoven, Faculteit Elektrotechniek, Postbus 513, 5600 MB Eindhoven, The Netherlands
}

Received February 26, 2004

\begin{abstract}
We analyze the evolution of the vortex and the asymmetrical parts of orbital angular momentum during its propagation through separable first-order optical systems. We find that the evolution of the vortex part depends on only parameters $a_{x}, a_{y}, b_{x}$, and $b_{y}$ of the ray transformation matrix and that isotropic systems with the same ratio $b / a$ produce the same change of the vortex part of the orbital angular momentum. Finally, it is shown that, when light propagates through an optical fiber with a quadratic refractive-index profile, the vortex part of the orbital angular momentum cannot change its sign more than four times per period. (C) 2004 Optical Society of America

OCIS codes: $\quad 070.2580,070.4690,030.5630,120.4820,140.3300$.
\end{abstract}

During the past decade the concept of orbital angular momentum (OAM) has been applied for the description of coherent optical vortex beams. ${ }^{1,2}$ In recent publications ${ }^{3,4}$ it was suggested that OAM be decomposed into two parts: the asymmetrical OAM and the vortex OAM. The first part describes an astigmatic beam but with a smooth wave front, while the second is related to the singularity of the wave front (screw dislocations).

In this Letter we study the evolution of the asymmetrical and the vortex OAM of linearly polarized, partially coherent beams during their propagation through separable first-order optical, or $A B C D$, systems. It was recently reported that some partially coherent fields also exhibit vortex behavior. ${ }^{5}$ Taking into account that the concept of OAM can be generalized to the case of partially coherent beams, ${ }^{6}$ one can apply the results of this study to both the completely coherent and the partially coherent cases.

We base the definition of OAM on the moments of the Wigner distribution (WD). ${ }^{7}$ The WD $W(x, u ; y, v)$ represents partially coherent light in a combined spacespatial-frequency domain, the so-called phase space, where $u$ is the spatial-frequency variable associated with space variable $x$ and $v$ is the spatial-frequency variable associated with space variable $y$. The treatment in this Letter is based on the normalized moments of the WD, where the normalization is with respect to total energy $E$ of the signal: $E=\iiint \int W(x, u ; y, v) \mathrm{d} x \mathrm{~d} u \mathrm{~d} y \mathrm{~d} v$. These normalized moments $\mu_{\text {pqrs }}$ of the WD are thus defined by

$$
\begin{aligned}
\mu_{p q r s} E= & \int_{-\infty}^{\infty} \int_{-\infty}^{\infty} \int_{-\infty}^{\infty} \int_{-\infty}^{\infty} W(x, u ; y, v) \\
& \times x^{p} u^{q} y^{r} v^{s} \mathrm{~d} x \mathrm{~d} u \mathrm{~d} y \mathrm{~d} v, \quad p, q, r, s \geq 0 .
\end{aligned}
$$

We restrict ourselves to the ten second-order moments, $p+q+r+s=2$, assuming moreover, without loss of generality, that the four first-order moments are zero.
OAM $\Lambda$ of an optical beam can be expressed in terms of second-order moments of its WD as [Eq. (3) of Ref. 4] $\Lambda=\left(E / c^{2}\right)\left(\mu_{1001}-\mu_{0110}\right)$. The asymmetrical part $\Lambda_{a}$ and the vortex part $\Lambda_{v}$ of the OAM can then be written as [Eqs. (22) and (21) of Ref. 4]

$$
\begin{aligned}
\Lambda_{a} & =\frac{E}{c^{2}} \\
& \times \frac{\left(\mu_{2000}-\mu_{0020}\right)\left(\mu_{1001}+\mu_{0110}\right)-2 \mu_{1010}\left(\mu_{1100}-\mu_{0011}\right)}{\mu_{2000}+\mu_{0020}},
\end{aligned}
$$

$$
\begin{aligned}
\Lambda_{v}= & \frac{2 E}{c^{2}} \\
& \times \frac{\mu_{0020} \mu_{1001}-\mu_{2000} \mu_{0110}+\mu_{1010}\left(\mu_{1100}-\mu_{0011}\right)}{\mu_{2000}+\mu_{0020}},
\end{aligned}
$$

respectively; note that we have $\Lambda_{a}+\Lambda_{v}=\Lambda$. It is easy to see that, for rotationally symmetric beams, for which $\mu_{2000}=\mu_{0020}$ and $\mu_{1010}=0,{ }^{8} \Lambda_{a}=0$. We note that $\Lambda_{v}$ is closely related to optical twist $T,{ }^{9}$ defined as

$$
T=\mu_{0020} \mu_{1001}-\mu_{2000} \mu_{0110}+\mu_{1010}\left(\mu_{1100}-\mu_{0011}\right) .
$$

To study the evolution of the WD moments in separable first-order systems, we note that the input-output relationship between the WD $W_{\text {in }}(x, u ; y, v)$ at the input plane and the WD $W_{\text {out }}(x, u ; y, v)$ at the output plane of a separable first-order optical system reads as ${ }^{10,11} W_{\text {out }}(x, u ; y, v)=$ $W_{\text {in }}\left(d_{x} x-b_{x} u,-c_{x} x+a_{x} u ; d_{y} y-b_{y} v,-c_{y} y+a_{y} v\right)$. This relationship is based on an affine transformation of the WD described by the so-called ray transformation matrix, ${ }^{12}$ or the $A B C D$ matrix. From the symplecticity condition ${ }^{12}$ of this ray transformation 
matrix we have, in the special case of a separable system, $a_{x} d_{x}-b_{x} c_{x}=1$ and $a_{y} d_{y}-b_{y} c_{y}=1$.

The normalized moments $\mu_{\text {pqu }}^{\text {out }}$ of the output WD $W_{\text {out }}(x, u ; y, v)$ are related to the normalized moments $\mu_{p q r s}^{\text {in }}=\mu_{p q r s}$ of the input WD $W_{\text {in }}(x, u ; y, v)$ as

$$
\begin{aligned}
\mu_{p q r s}^{\text {out } E}= & \int_{-\infty}^{\infty} \int_{-\infty}^{\infty} \int_{-\infty}^{\infty} \int_{-\infty}^{\infty} W_{\text {in }}(x, u ; y, v) \\
& \times\left(a_{x} x+b_{x} u\right)^{p}\left(c_{x} x+d_{x} u\right)^{q} \\
& \times\left(a_{y} y+b_{y} v\right)^{r}\left(c_{y} y+d_{y} v\right)^{s} \mathrm{~d} x \mathrm{~d} u \mathrm{~d} y \mathrm{~d} v \\
= & E \sum_{k=0}^{p} \sum_{l=0}^{q} \sum_{m=0}^{r} \sum_{n=0}^{s}\left(\begin{array}{c}
p \\
k
\end{array}\right)\left(\begin{array}{c}
q \\
l
\end{array}\right)\left(\begin{array}{c}
r \\
m
\end{array}\right)\left(\begin{array}{c}
s \\
n
\end{array}\right) \\
& \times a_{x}^{p-k} b_{x}^{k} c_{x}^{l} d_{x}^{q-l} a_{y}^{r-m} b_{y}^{m} c_{y}^{n} d_{y}^{s-n} \\
& \times \mu_{p-k+l, q-l+k, r-m+n, s-n+m} .
\end{aligned}
$$

We note that, for Fourier-transform systems, with respect to one or two variables, the output moments are related to the input moments by the simple relationships

$$
\begin{aligned}
\left.\mu_{p q r s}^{\text {out }}\right|_{\mathrm{FT}_{x}} & =\mu_{q p r s}(-1)^{q} b_{x}^{p-q}, \\
\left.\mu_{p q r s}^{\text {out }}\right|_{\mathrm{FT}_{y}} & =\mu_{p q s r}(-1) b_{y}^{r-s}, \\
\left.\mu_{p q r s}^{\text {out }}\right|_{\mathrm{FT}_{x y}} & =\mu_{q p s r}(-1)^{q+s} b_{x}^{p-q} b_{y}^{r-s},
\end{aligned}
$$

where $\mathrm{FT}_{x}, \mathrm{FT}_{y}$, and $\mathrm{FT}_{x y}$ represent the Fourier transforms with respect to $x$ (with parameters $a_{x}=d_{x}=0$, $b_{x} c_{x}=-1, a_{y}=d_{y}=1, b_{y}=c_{y}=0$ ), to $y$ (with parameters $a_{x}=d_{x}=1, b_{x}=c_{x}=0, a_{y}=d_{y}=0, b_{y} c_{y}=-1$ ), and to both $x$ and $y$ (with parameters $a_{x}=d_{x}=0$, $\left.b_{x} c_{x}=-1, a_{y}=d_{y}=0, b_{y} c_{y}=-1\right)$, respectively. If we combine these simple relationships [Eqs. (6)] with the definition of the twist [Eq. (4)], we can readily derive the twists, $\left.T^{\text {out }}\right|_{\mathrm{FT}_{x}}=b_{x} T_{x},\left.T^{\text {out }}\right|_{\mathrm{FT}_{y}}=b_{y} T_{y}$, and $\left.T^{\text {out }}\right|_{\mathrm{FT}_{x y}}=b_{x} b_{y} T_{x y}$, at the output of these respective Fourier-transform systems, and we have

$$
\begin{gathered}
T_{x}=\mu_{0020} \mu_{0101}+\mu_{0200} \mu_{1010}-\mu_{0110}\left(\mu_{1100}+\mu_{0011}\right), \\
T_{y}=-\mu_{0002} \mu_{1010}-\mu_{2000} \mu_{0101}+\mu_{1001}\left(\mu_{1100}+\mu_{0011}\right), \\
T_{x y}=-\mu_{0002} \mu_{0110}+\mu_{0200} \mu_{1001}-\mu_{0101}\left(\mu_{1100}-\mu_{0011}\right) .
\end{gathered}
$$

To study in particular the evolution of the twist and the OAM, we first observe that there are seven relevant second-order moments that make up the vortex [Eq. (2)] and the asymmetrical [Eq. (3)] parts of the OAM; the purely $u v$ moments (i.e., $\mu_{0200}, \mu_{0002}$, and $\mu_{0101}$, for which $p=r=0$ ) do not enter into Eqs. (2) and (3). Moment relationship (5) immediately leads to the necessary expressions that describe the values of these seven moments in the output of an $A B C D$ system in terms of the ten second-order input moments. In particular, OAM $\Lambda^{\text {out }}=\left(E / c^{2}\right)\left(\mu_{1001}^{\text {out }}-\mu_{0110}^{\text {out }}\right)$ at the output of a separable $A B C D$ system follows from

$$
\begin{aligned}
\mu_{1001}^{\text {out }}= & a_{x} c_{y} \mu_{1010}+a_{x} d_{y} \mu_{1001} \\
& +b_{x} c_{y} \mu_{0110}+b_{x} d_{y} \mu_{0101}, \\
\mu_{0110}^{\text {out }}= & c_{x} a_{y} \mu_{1010}+c_{x} b_{y} \mu_{1001} \\
& +d_{x} a_{y} \mu_{0110}+d_{x} b_{y} \mu_{0101}
\end{aligned}
$$

and takes the form

$$
\begin{aligned}
\Lambda^{\text {out }}= & \frac{E}{c^{2}}\left[\mu_{1001}\left(a_{x} d_{y}-b_{y} c_{x}\right)-\mu_{0110}\left(a_{y} d_{x}-b_{x} c_{y}\right)\right. \\
& \left.+\mu_{0101}\left(b_{x} d_{y}-b_{y} d_{x}\right)+\mu_{1010}\left(a_{x} c_{y}-a_{y} c_{x}\right)\right] .
\end{aligned}
$$

To obtain the evolution of the vortex and asymmetrical parts of the OAM, let us first consider output twist $T^{\text {out }}$. After a straightforward but rather lengthy calculation, using the definition of the twist [Eq. (4)], moment relationship (5), and definitions (7), it can be shown that the following expression holds for the output twist:

$$
T^{\mathrm{out}}=a_{x} a_{y} T+a_{y} b_{x} T_{x}+a_{x} b_{y} T_{y}+b_{x} b_{y} T_{x y} .
$$

Further, we need the expression

$$
\begin{aligned}
\mu_{2000}^{\text {out }}+\mu_{0020}^{\text {out }}= & a_{x}^{2} \mu_{2000}+2 a_{x} b_{x} \mu_{1100}+b_{x}^{2} \mu_{0200} \\
& +a_{y}^{2} \mu_{0020}+2 a_{y} b_{y} \mu_{0011}+b_{y}^{2} \mu_{0002}
\end{aligned}
$$

for the denominator that appears in Eqs. (2) and (3). Note that the denominator is always positive, which is a direct consequence of the fact that moments $\mu_{2000}$ and $\mu_{0020}$ (and also $\mu_{0200}$ and $\mu_{0002}$ ) are positive by definition. From Eqs. (9) and (10), we immediately conclude that the evolution of vortex part $\Lambda_{v}$ through a separable $A B C D$ system depends on only parameters $a_{x}$, $a_{y}, b_{x}$, and $b_{y}$ of the ray transformation matrix. This conclusion also implies that $\Lambda_{v}$ cannot be changed by a quadratic phase corrector ${ }^{3,4}\left(b_{x}=b_{y}=0, a_{x}=a_{y}=1\right)$; note that in this case $\Lambda^{\text {out }}=\Lambda+\left(E / c^{2}\right) \mu_{1010}\left(c_{y}-c_{x}\right)$.

For an isotropic system, i.e., $a_{x}=a_{y}, b_{x}=b_{y}$, $c_{x}=c_{y}$, and $d_{x}=d_{y}$, general equation (8) reduces to $\Lambda^{\text {out }}=\left(E / c^{2}\right)\left(\mu_{1001}-\mu_{0110}\right)=\Lambda$, and we conclude that the OAM does not change when the beam propagates through an isotropic $A B C D$ system. However, this property does not hold for the vortex and the asymmetrical parts of the OAM, as was demonstrated for free-space propagation. ${ }^{3,4}$ In the special case of an isotropic system, Eqs. (9) and (10) reduce to simpler forms and we get

$$
\Lambda_{v}^{\text {out }}=\frac{2 E}{c^{2}} \frac{a^{2} T+a b\left(T_{x}+T_{y}\right)+b^{2} T_{x y}}{a^{2}\left(\mu_{2000}+\mu_{0020}\right)+2 a b\left(\mu_{1100}+\mu_{0011}\right)+b^{2}\left(\mu_{0200}+\mu_{0002}\right)} .
$$


We note that, for the special signal for which the relation

$$
\frac{T}{\mu_{2000}+\mu_{0020}}=\frac{T_{x}+T_{y}}{2\left(\mu_{1100}+\mu_{0011}\right)}=\frac{T_{x y}}{\mu_{0200}+\mu_{0002}}
$$

holds, the vortex part of its OAM does not change in isotropic systems. This holds in particular for rotationally symmetric beams, for which $\mu_{0020}=\mu_{2000}$, $\mu_{0002}=\mu_{0200}, \mu_{0011}=\mu_{1100}, \mu_{0110}=-\mu_{1001}$, and $\mu_{1010}=\mu_{0101}=0,{ }^{8}$ and hence $T=2 \mu_{2000} \mu_{1001}$, $T_{x}=T_{y}=2 \mu_{1100} \mu_{1001}$, and $T_{x y}=2 \mu_{0200} \mu_{1001}$, and for which the three expressions in Eq. (12) thus take the value $\mu_{1001}$ : A rotationally symmetric beam propagating through an isotropic system does not change its vortex, and the asymmetrical part of its OAM remains zero.

We note that, for isotropic systems with $b=0$ (and $a d=1$ ), the vortex part of the OAM (as well as the asymmetrical part of it) is preserved: $\Lambda_{v}^{\text {out }}=\Lambda_{v}$ and $\Lambda_{a}^{\text {out }}=\Lambda_{a}$. This is the case for all systems for which the input and output planes are conjugate planes.

Let us now study general expression (11) for the evolution of the vortex part of the OAM, which we may as well express in the form

$$
\begin{aligned}
& \Lambda_{v}^{\text {out }}=\Lambda_{v}(p)=\frac{2 E}{c^{2}} \\
& \times \frac{T+p\left(T_{x}+T_{y}\right)+p^{2} T_{x y}}{\left(\mu_{2000}+\mu_{0020}\right)+2 p\left(\mu_{1100}+\mu_{0011}\right)+p^{2}\left(\mu_{0200}+\mu_{0002}\right)},
\end{aligned}
$$

where we introduce the ratio $p=b / a$. Optical systems with the same value of $p$ behave similarly with respect to the evolution of $\Lambda_{v}$. As examples we mention (i) a section of free space in the paraxial approximation, or parabolic system ${ }^{13}$ (with $a=d=1, c=0$, $p=b$, and $b$ proportional to the propagation distance), (ii) a fractional Fourier-transform system, ${ }^{14}$ or elliptic system ${ }^{13}$ (with $a=d=\cos \alpha, b=-c=\sin \alpha$, and $p=\tan \alpha$ ), and (iii) a hyperbolic system ${ }^{13}$ (with $a=d=\cosh \alpha, b=c=\sinh \alpha$, and $p=\tanh \alpha$ ). Analyzing Eq. (13), one can find the values of $p$ where $\Lambda_{v}(p)=0$ or has maxima or minima. Note that, while the denominator is positive for all possible values of $p$, the numerator, and therefore $\Lambda_{v}(p)$ itself, may or may not change its sign (but no more than twice), depending on the actual values of $T, T_{x}+T_{y}$, and $T_{x y}$. Clearly, $\Lambda_{v}(p)$ takes the value zero for those values of $p$ for which $p=\left\{-\left(T_{x}+T_{y}\right) \pm\left[\left(T_{x}+T_{y}\right)^{2}-\right.\right.$ $\left.\left.4 T T_{x y}\right]^{1 / 2}\right\} / 2 T_{x y}$, and since $p$ has to be real, this is possible only if the condition $\left(T_{x}+T_{y}\right)^{2} \geq 4 T T_{x y}$ holds.

In the case of free-space propagation, for which $p$ is associated with the propagation distance, only positive values of $p$ are allowed, and $\Lambda_{v}(0)=\left(2 E / c^{2}\right) T /\left(\mu_{2000}+\right.$ $\left.\mu_{0020}\right)$ and $\Lambda_{v}(p \rightarrow \infty)=\left(2 E / c^{2}\right) T_{x y} /\left(\mu_{0200}+\mu_{0002}\right)$; note that the case $p \rightarrow \infty$ corresponds to Fourier transformation. Hence, for $T T_{x y}<0$, there is only one posi- tive solution where $\Lambda(p)$ changes its sign. On the other hand, for $T T_{x y}>0$, there are two positive solutions if the signs of $T_{x y}$ and $T_{x}+T_{y}$ are different, and there are no positive solutions if these signs are the same. Moreover, in the special case that the initial field (or its Fourier transform) is vortex free, $T=0$ (or $T_{x y}=0$ ), vortex $\Lambda_{v}(p)$ also takes the value zero for $p=-\left(T_{x}+T_{y}\right) / T_{x y}$, which is positive if $T_{x y}$ and $T_{x}+T_{y}$ have different signs [or for $p=-T /\left(T_{x}+T_{y}\right)$, which is positive if $T$ and $T_{x}+T_{y}$ have different signs].

In the case of a fractional Fourier-transform system, in which $p$ is associated with fractional angle $\alpha$ and $p=\tan \alpha$, both positive and negative values of $p$ are acceptable. Positive values of $p$ correspond to the first and third quadrants of angle $\alpha$, and negative values correspond to the second and fourth quadrants. Therefore, during one period $0 \leq \alpha \leq 2 \pi$, the sign of $\Lambda_{v}(p)$ changes four times if $\left(T_{x}+T_{y}\right)^{2}>4 T T_{x y}$, twice if $\left(T_{x}+T_{y}\right)^{2}=4 T T_{x y}$, and does not change if $\left(T_{x}+T_{y}\right)^{2}<4 T T_{x y}$. Note that this analysis is useful for understanding the propagation of vortex beams through optical fibers with quadratic refractive-index profiles, since in the paraxial approximation of the scalar diffraction theory the complex field amplitude of monochromatic light propagating through such a fiber is fractionally Fourier transformed, with angle $\alpha$ being proportional to the propagation distance in the fiber. ${ }^{15,16}$

T. Alieva thanks the Spanish Ministry of Science and Technology for financial support of this research (Ramon y Cajal grant and project TIC 2002-01846). M. J. Bastiaan's e-mail address is m.j.bastiaans@tue.nl. T. Alieva's e-mail address is talieva@fis.ucm.es.

\section{References}

1. M. S. Soskin, V. N. Gorshkov, M. V. Vasnetsov, J. T. Malos, and N. R. Heckenberg, Phys. Rev. A 56, 163 (1997).

2. M. S. Soskin and M. V. Vasnetsov, Prog. Opt. 42, 219 (2001).

3. A. Ya. Bekshaev, M. V. Vasnetsov, V. G. Denisenko, and M. S. Soskin, JETP Lett. 75, 127 (2002).

4. A. Ya. Bekshaev, M. S. Soskin, and M. V. Vasnetsov, J. Opt. Soc. Am. A 20, 1635 (2003).

5. H. F. Schouten, G. Gbur, T. D. Visser, and E. Wolf, Opt. Lett. 28, 968 (2003).

6. J. Serna and J. M. Movilla, Opt. Lett. 26, 405 (2001).

7. M. J. Bastiaans, Opt. Commun. 25, 26 (1978).

8. M. J. Bastiaans and T. Alieva, Opt. Lett. 28, 2443 (2003).

9. M. J. Bastiaans, J. Opt. Soc. Am. A 17, 2475 (2000).

10. M. J. Bastiaans, J. Opt. Soc. Am. 69, 1710 (1979).

11. M. J. Bastiaans, J. Opt. Soc. Am. A 3, 1227 (1986).

12. R. K. Luneberg, Mathematical Theory of Optics (University of California Press, Berkeley, Calif., 1966).

13. K. B. Wolf, Integral Transforms in Science and Engineering (Plenum, New York, 1979), Chap. 9.

14. A. W. Lohmann, J. Opt. Soc. Am. A 10, 2181 (1993).

15. G. P. Agrawal, A. K. Ghatak, and C. L. Metha, Opt. Commun. 12, 333 (1974).

16. D. Mendlovic and H. M. Ozaktas, J. Opt. Soc. Am. A 10, 1875 (1993). 